\title{
Critical closing pressure in cerebrovascular circulation
}

Marek Czosnyka, Piotr Smielewski, Stefan Piechnik, Pippa G Al-Rawi, Peter J Kirkpatrick, Basil F Matta, John D Pickard

\begin{abstract}
Objective-Cerebral critical closing pressure (CCP) has been defined as an arterial pressure threshold below which arterial vessels collapse. Hypothetically this is equal to intracranial pressure (ICP) plus the contribution from the active tone of cerebral arterial smooth muscle. The correlation of CCP with ICP, cerebral autoregulation, and other clinical and haemodynamic modalities in patients with head injury was evaluated.

Method-intracranial pressure, arterial blood pressure (ABP) and middle cerebral artery blood flow velocity were recorded daily in ventilated patients. Waveforms were processed to calculate CCP, the transcranial Doppler-derived cerebral autoregulation index $(\mathbf{M x})$, mean arterial pressure $(\mathrm{ABP})$, intracranial pressure (ICP), and cerebral perfusion pressure (CPP).
\end{abstract}

Results-Critical closing pressure reflected the time related changes in ICP during plateau and $B$ waves. Overall correlation between CCP and ICP was mild but significant $(R=0.41 ; p<0.0002)$. The mean difference between ABP and CCP correlated with CPP $(R=0.57,95 \%$ confidence interval (95\% CI) for prediction $25 \mathrm{~mm} \mathrm{Hg}$ ). The difference between CCP and ICP, described previously as proportional to arterial wall tension, correlated with the index of cerebral autoregulation $\mathrm{Mx} \quad(\mathrm{p}<0.0002)$ and $\mathrm{CPP}$ $(p<0.0001)$. However, by contrast with the Mx index, CCP-ICP was not significantly correlated with outcome after head injury. Conclusion-Critical closing pressure, although sensitive to variations in ICP and CPP, cannot be used as an accurate estimator of these modalities with acceptable confidence intervals. The difference CCP-ICP significantly correlates with cerebral autoregulation, but it lacks the power to predict outcome after head injury.

(F Neurol Neurosurg Psychiatry 1999;66:606-611)

Keywords: critical closing pressure; cerebral autoregulation; head injury; transcranial Doppler

The versatility of transcranial Doppler ultrasonography (TCD) has encouraged imaginative applications in intensive care of patients with head injury. Although the blood flow velocity (FV) cannot express baseline volume flow, ${ }^{1-3}$ the dynamic changes of $\mathrm{CBF}$ are usually reflected in the TCD readings. ${ }^{1}$ The response of FV to a critical decrease in CPP is sensitive, and usually immediate. Different methods for continuous analysis of TCD waveform have been reported before, such as analysis of pulsatility index, ${ }^{4}$ vascular impedance, ${ }^{56}$ and slow waves. $^{7-9}$

The concept of "critical closing pressure" (CCP) was first described around 50 years ago by Burton. ${ }^{10} \mathrm{He}$ developed a theoretical model demonstrating that small vessels can collapse when the arterial pressure approaches a critical value, defined as CCP. In the cerebral circulation this value, according to Burton's model, should be equal to the sum of intracranial pressure (ICP) and a component proportional to the active tension of vascular smooth muscle: ${ }^{11}$

$\mathrm{CCP}=\mathrm{ICP}+$ tension of arterial walls

More recently a method for the calculation of CCP using transcranial Doppler ultrasonography has been proposed by Aaslid. ${ }^{12} \mathrm{He}$ suggested that the intercept point of a regression line between arterial systolic and diastolic pressure plotted along the $\mathrm{x}$ axis and the systolic and diastolic values of blood flow velocity in the middle cerebral artery plotted along the $\mathrm{y}$ axis, can be used for the estimation of CCP(fig 1). Michel et $a l^{13}$ have recently presented an alternative method for the calculation of CCP, based on the first harmonics of the pulse waveforms of arterial pressure and blood flow velocity.

However, this promising and non-invasive methodology has not yet been fully evaluated. The studies reported by Panerai et $a l^{1415}$ and Michel et $a l^{16}$ in newborn babies have not explored the correlation of CCP with ICP or clinical variables, except to postulate that CCP may be useful for monitoring the cerebral haemodynamics or the state of autoregulation.

Our aim was to study CCP in adult patients with head injury. In particular, the following questions were considered:

What is the typical reaction of CCP to intracranial hypertension and arterial hypotension or hypertension?

Does CCP depend on cerebral autoregulation, cerebrovascular resistance, and other established indices of cerebral haemodynamics?

How does CCP compare with the severity of injury and outcome?

\section{Materials and methods}

PATIENTS

Ninety eight patients with head injury admitted to the neurointensive care unit in Addenbrooke's Hospital with a mean Glasgow coma in revised form

7 August 1998

Accepted 25 August 1998 

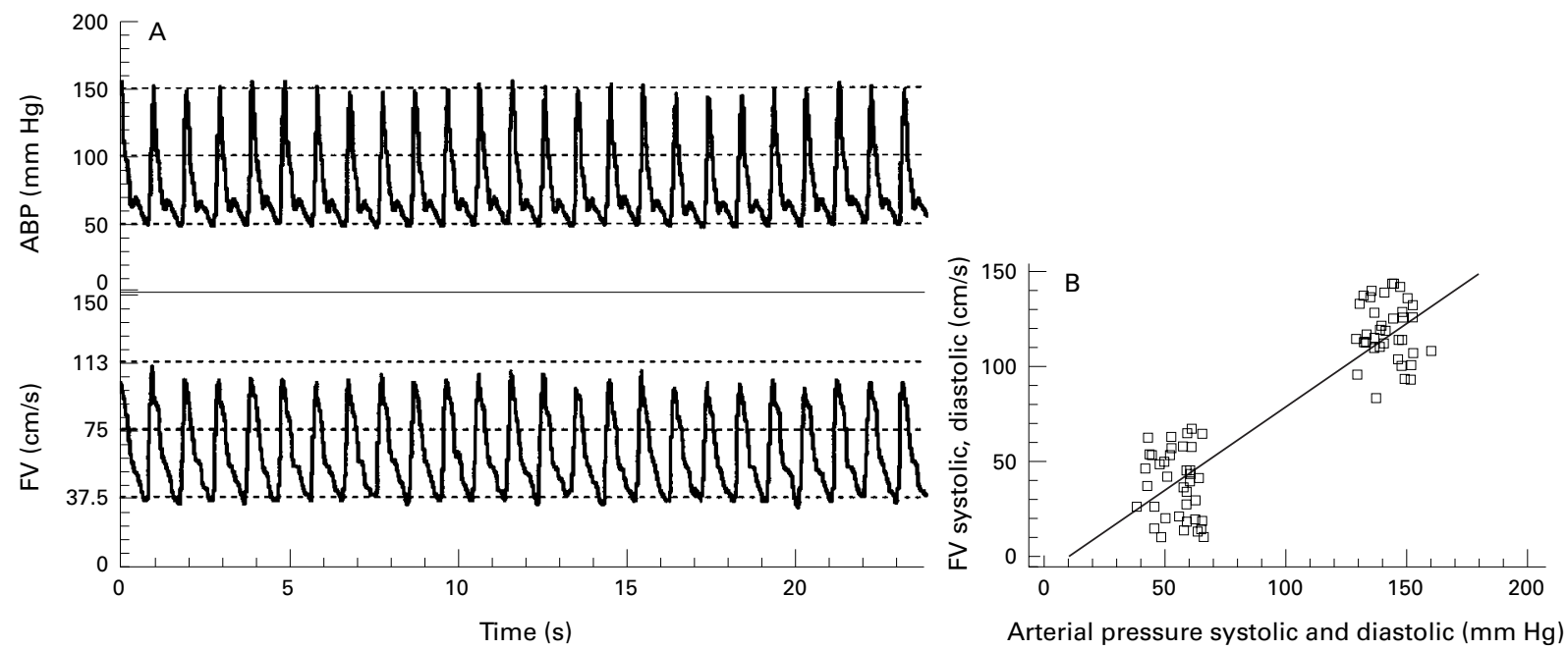

Figure 1 Critical closing pressure may be calculated as an intercept point of averaged consecutive systolic and diastolic values of $(A)$ arterial blood pressure $(A B P)$ and flow velocity $(F V)$ calculated from a limited time period, $(B)$ plotted on $x-y$ plane.

score $<8$ (range of admission score was 3 to 13 ) were studied. There were 30 females and 68 males, age range 14-76 years (mean age 38 years). All patients were paralysed, sedated, and ventilated to achieve mild hypocapnia. Alternating colloid and normal saline infusions, with addition of inotropic agents (infusion of dopamine with a rate of $2-15$ $\mathrm{g} / \mathrm{kg} /$ minute) were used selectively to prevent arterial hypotension causing reduction of cerebral perfusion pressure $(\mathrm{CPP})<60 \mathrm{~mm} \mathrm{Hg}$. Boluses of mannitol $(200 \mathrm{ml}$ of $20 \%$, over a period of 20 minutes or longer) were given to manage rising intracranial pressure $>25 \mathrm{~mm}$ $\mathrm{Hg}$.

Outcome was assessed at 6 months after injury.

MONITORING

Arterial pressure (ABP) was monitored directly in the radial or dorsalis pedis artery (System 8000, S\&W Vickers Ltd, Sidcup, UK). Intracranial pressure (ICP) was monitored continuously using a fibreoptic transducer (Camino Direct Pressure Monitor, Camino Laboratories, San Diego, CA, USA). The catheter tip was inserted into the frontal region intraparenchymally.

The middle cerebral artery (MCA) blood flow velocity was measured (PCDop 842 Doppler Ultrasound Unit (Scimed, Bristol, UK; used in 86) or Neuroguard (Medasonics, Fremont, CA, USA; used in 10 patients) transcranial ultrasonographs) daily for a period from 20 minutes to 4 hours starting from the day of admission either until discharge or day 8 after head injury. The reason for using material acquired with two different ultrasonographs can be justified by laboratory assessment (four healthy volunteers) where no difference in averaged systolic/diastolic blood flow velocity recorded using both machines was found, exceeding variability described in the literature. ${ }^{17}$ It is worthwhile mentioning that PCDop 842 is unable to measure flow velocity $>200 \mathrm{~cm} / \mathrm{s}$. The limitation for Neuroguard is $400 \mathrm{~cm} / \mathrm{s}$. Therefore the PCDop 842 is not recommended for use in patients in whom cerebral vasospasm is suspected. In fact, with PCDop 842 a the depth of insonation was always adjusted to obtain an undistorted waveform (avoiding sections of potentially spasmic MCA in which momentary flow velocity exceeded $200 \mathrm{~cm} / \mathrm{s}$ ). It seems to be acceptable for studying CCP, as vasospasm affects pressure-flow conditions in the basal arteries to an extent which is difficult to estimate without precise and invasive measurement. ${ }^{3}$

The recordings were undertaken during periods of stable respiration undisturbed by physiotherapy, tracheal suction, or other nursing interventions.

DATA ANALYSIS

Analogue outputs from the pressure monitors and the TCD ultrasonograph (maximal frequency envelope was used) were connected to an IBM PC laptop computer (Amstrad ALT 386 SX, UK) fitted with an analogue to digital converter (DT 2814, Data Translation, Marlboro, USA). Signals were sampled at a rate of 50 $\mathrm{Hz}$ and stored on the hard disk using specific software for the waveform recording (W Zabolotny, Warsaw University of Technology, Poland). Digital signals were then processed off line using software developed in house (MC). Over 450 recordings were analysed.

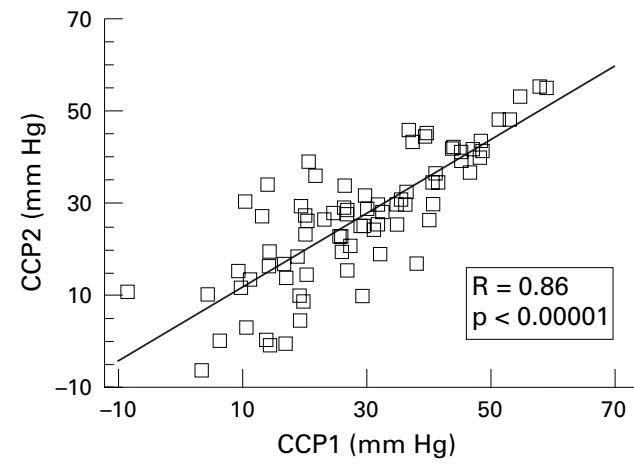

Figure 2 Correlation plot between CCP calculated from systolic and diastolic values of $A B P$ and $F V$ (CCP: $y$ axis) and from first harmonics of pulse waves of $A B P$ and $F V$ waveforms (CPP1: $x$ axis). 

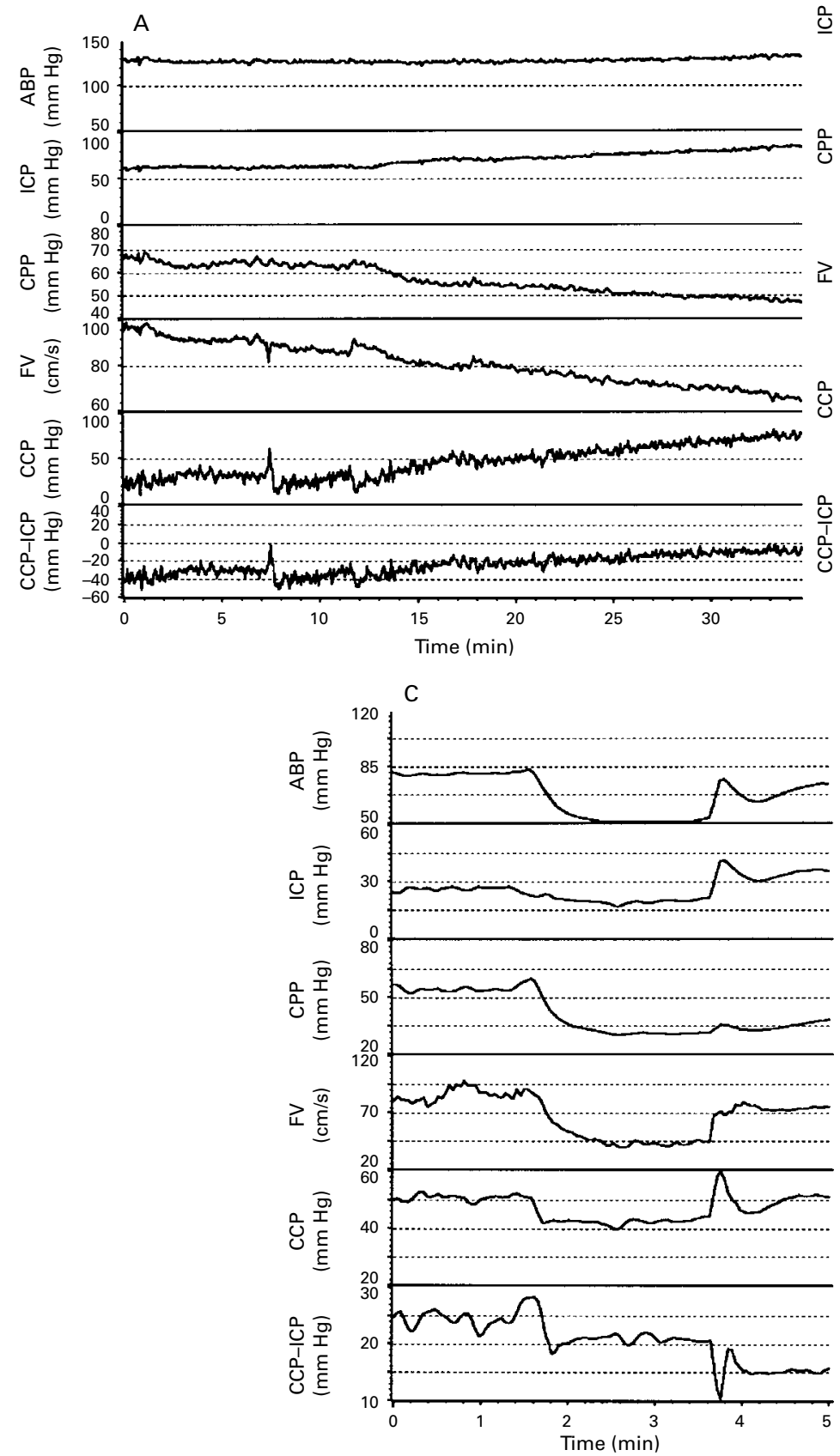
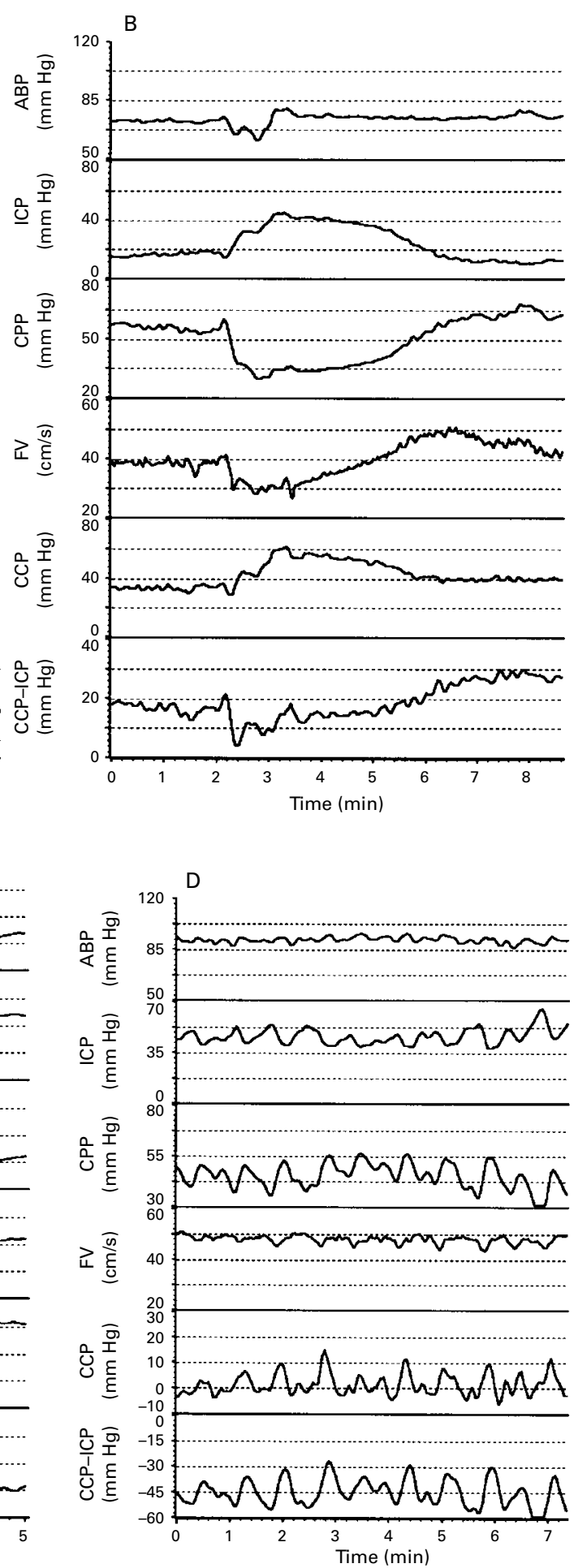

Figure 3 (A) Examples of time variability of mean arterial pressure (ABP), intracranial pressure (ICP), cerebral perfusion pressure (CPP), mean flow velocity (FV), critical closing pressure (CCP), and the difference between CCP and ICP recorded during terminal increase in ICP; (B) ICP plateau wave; (C) incidental arterial hypotension; (D) B waves of ICP.

Time averaged values of ICP, ABP, CPP, $(\mathrm{CPP}=\mathrm{ICP}-\mathrm{ABP})$, and MCA blood flow velocity $(\mathrm{FV})$ were calculated using waveform time integration over 6 second intervals. From the same periods flow velocity systolic and diastolic (FVs and FVd), arterial blood pressure systolic and diastolic (ABPs, ABPd) as well as the fundamental harmonics of pulse waves of arterial pressure and flow velocity waveforms
(A1 and F1 respectively) were calculated. The fast Fourier transform algorithm was used to calculate the power spectra of the waveforms.

Critical closing pressure was calculated from the 6 second intervals using two different methods:

$\mathrm{CCP} 1=\mathrm{ABPs}-(\mathrm{ABPs}-\mathrm{ABPd}) /(\mathrm{FVs}-\mathrm{FVd}) \times$ FVs (fig 1)

$\mathrm{CCP} 2=\mathrm{ABP}-\mathrm{A} 1 / \mathrm{F} 1 \times \mathrm{FV}$ 
Rank correlation coefficients between parameters studied in 98 patients with head injury

\begin{tabular}{|c|c|c|c|c|c|c|c|c|c|}
\hline & $C C P$ & $C C P-I C P$ & $A B P-C C P$ & $I C P$ & $C P P$ & $A B P$ & $F V$ & $M x$ & GOS \\
\hline CCP-ICP & $0.68^{\star}$ & & & & & & & & \\
\hline ABP-CCP & $-0.66^{\star}$ & $-0.505^{\star}$ & & & & & & & \\
\hline ICP & $0.41^{\star}$ & $-0.29^{\star}$ & -0.22 & & & & & & \\
\hline $\mathrm{CPP}$ & 0.05 & $0.52^{\star}$ & $0.38^{\star}$ & $-0.61^{\star}$ & & & & & \\
\hline $\mathrm{ABP}$ & $0.5^{\star}$ & $0.34^{\star}$ & 0.223 & 0.21 & $0.60^{\star}$ & & & & \\
\hline $\mathrm{FV}$ & -0.19 & -0.21 & $0.304^{\star}$ & -0.05 & 0.085 & 0.097 & & & \\
\hline $\mathrm{Mx}$ & 0.08 & $-0.25^{\star}$ & -0.05 & $0.45^{\star}$ & $-0.34^{\star}$ & 0.006 & 0.051 & & \\
\hline GOS & 0.13 & -0.032 & 0.0 & 0.17 & -0.031 & 0.15 & -0.06 & $0.39^{\star}$ & \\
\hline GCS & -0.02 & 0.109 & 0.13 & -0.09 & 0.17 & 0.11 & -0.02 & $-0.28^{\star}$ & $-0.41^{\star}$ \\
\hline
\end{tabular}

$\mathrm{CCP}=$ critical closing pressure (calculated using first harmonic components of pulse waveforms of blood flow velocity and arterial pressure); $\mathrm{ICP}=$ intracranial pressure; $\mathrm{ABP}=$ arterial pressure; $\mathrm{CPP}=$ cerebral perfusion pressure; $\mathrm{FV}=\mathrm{blood}$ flow velocity in the middle cerebral artery; $\mathrm{Mx}=$ autoregulation index ${ }^{9}$, calculated as running (over 4 minutes) correlation coefficient between slow waves in FV and CPP; GOS- outcome, dichotomised 1- favourable, 2-unfavourable; GCS=Glasgow coma score on admission.

* Correlation significant at $\mathrm{p}<0.05$; multiple comparison correction has been taken into account.

The second formula, recently postulated by Michel et $a l^{13}$ uses the first harmonics of ABP and FV pulse waveforms, rather than diastolic/ systolic values.

Pearson's moving correlation coefficient between FV and CPP using 40 consecutive averages $(\mathrm{Mx})$ was computed every 6 seconds; $\mathrm{Mx}$ has been previously reported to characterise cerebral autoregulation. ${ }^{9}$ A positive value of $\mathrm{Mx}$ signifies a positive association between slow waves of FV and CPP, which can be interpreted as a disturbed autoregulation, whereas zero or negative values of $\mathrm{Mx}$ have been shown to signify good autoregulation.
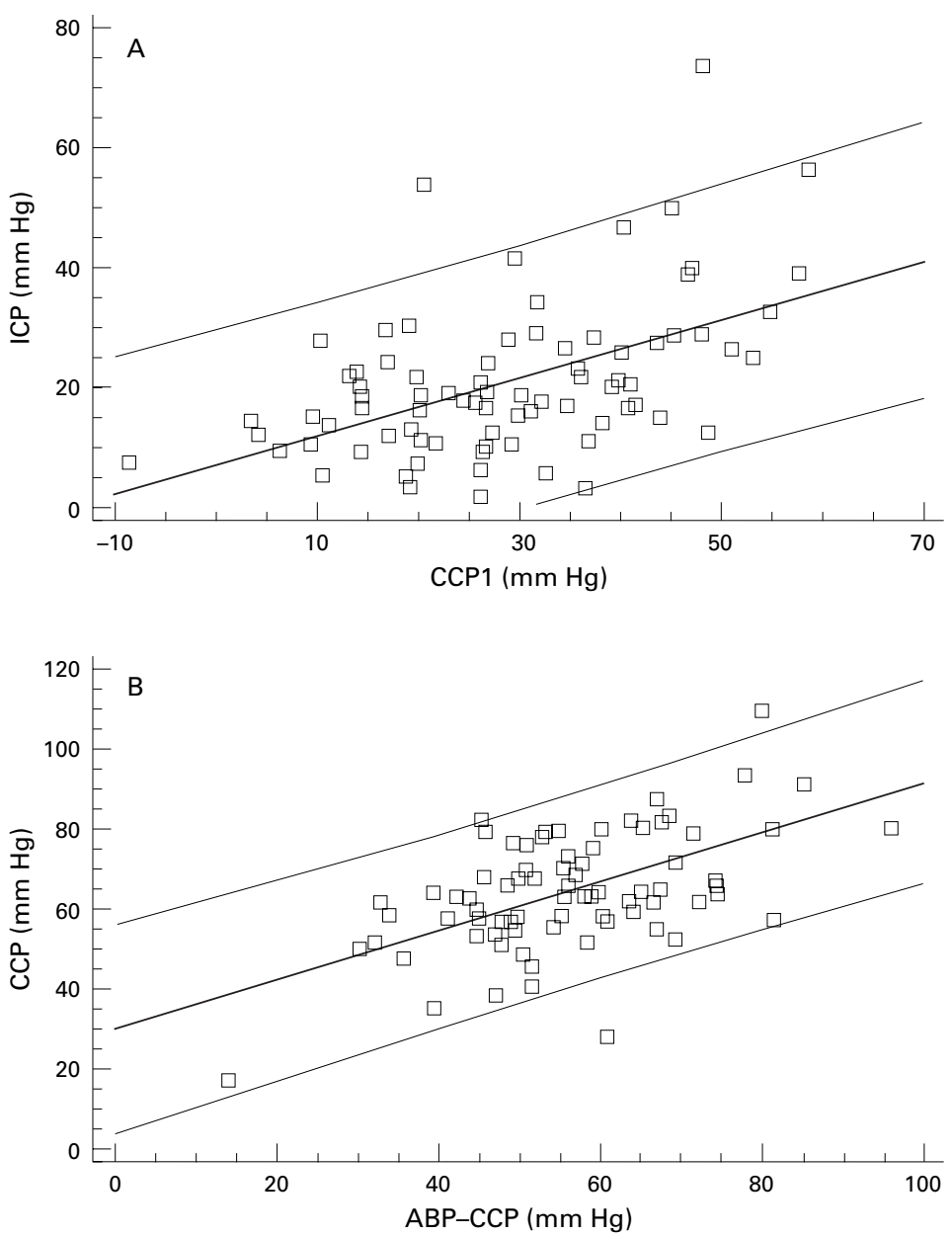

Figure 4 (A) Regression relation between $C C P 1$ and $I C P ; R=0.51 ; p<0.00001$, and $(B)$ difference between $A B P$ and $C C P 1$ and $C P P ; R=0.58 ; p<0.00001 .95 \% C I$ for prediction ranges $25 \mathrm{~mm} \mathrm{Hg}$.
For statistical analysis, data from every recording were time averaged and then averaged again for multiple recordings performed in the same patients, hence producing one averaged value per patient.

Rank correlation coefficients were calculated to investigate the statistical significance of associations between different variables; correction for multiple comparisons has been performed. Analysis of variance (ANOVA) and simple regression were used to illustrate the nature of the relation between selected variables.

\section{Results}

CALCULATION OF CCP

The mean value of CCP1 was $26 \mathrm{~mm} \mathrm{Hg}$ and for CCP2 it was $28 \mathrm{~mm} \mathrm{Hg}$; the difference was not significant. The correlation coefficient between CCP1 and CCP2 was 0.86 and was highly significant $\left(\mathrm{p}<10^{-6}\right)$. The dependence between CCP1 and CCP2 was best described by a linear model (fig 2). The absolute difference between CCP1 and CCP2 was independent of the state of autoregulation, outcome, ICP, CPP, or FV. The difference decreased significantly with age $(R=-0.26$; $\mathrm{p}<0.05)$. The method for CCP calculation based on fundamental components of $\mathrm{FV}$ and $\mathrm{ABP}$ waveforms (CPP2) was used for further analysis and denoted for simplicity as CCP.

TIME RELATED CHANGES OF CCP: CLINICAL OBSERVATIONS

During refractory intracranial hypertension $(n=3)$ increases in both CCP and the difference CCP-ICP were directly related to increase in ICP (fig 3A).

During plateau waves of ICP ( $\mathrm{n}=8$ recordings) CCP followed the pattern of ICP whereas the difference CCP-ICP decreased (fig 3B).

Arterial hypotension (fig 3C) seemed to decrease CCP. This was not always associated with a decrease in ICP, as in some instances ICP increased. The difference CCP-ICP also decreased but not as consistently.

Both CCP and CCP-ICP replicated $\mathrm{B}$ waves seen in ICP (fig 3D).

CORRELATION WITH OTHER VARIABLES AND

CLINICAL PARAMETERS

The table summarises correlations between CCP, CCP-ICP, ABP-CCP, and other parameters studied in this group of patients. 

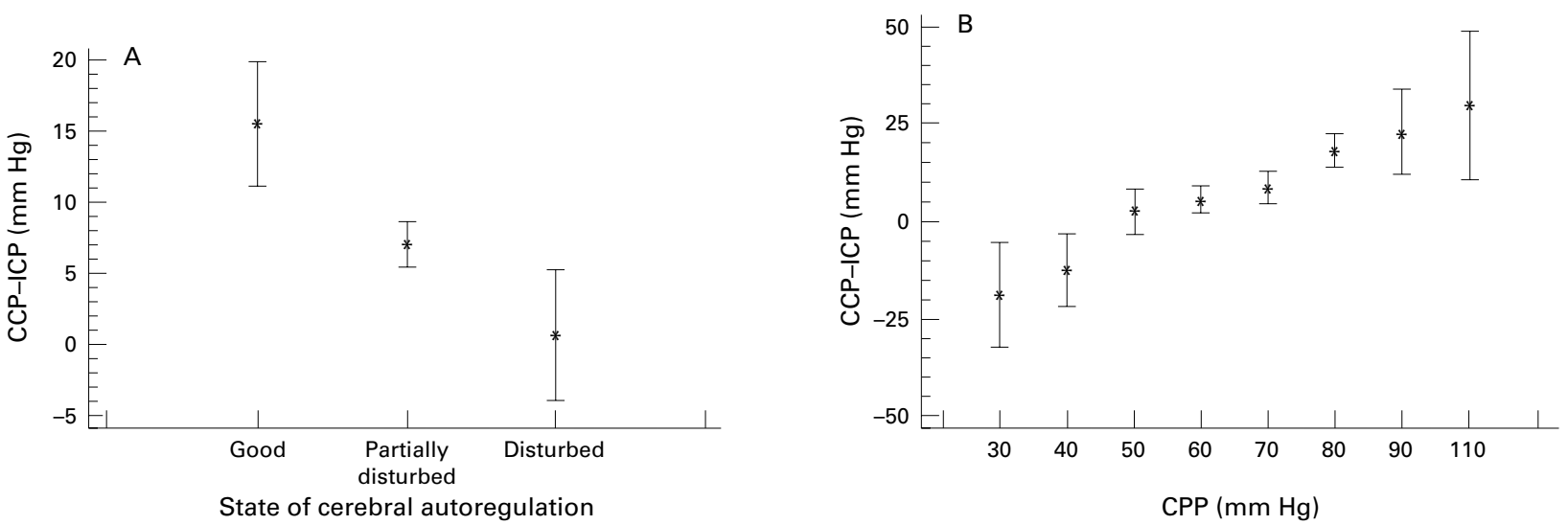

Figure 5 ANOVA plots (vertical bars-95\% CIs) of $(A)$ the difference between CCP and ICP and (B) the state of cerebral autoregulation and CPP.

The relation between CCP and ICP, although significant, indicated that CCP is an inaccurate estimator of ICP (fig 4), with 95\% CI for predictors of $27 \mathrm{~mm} \mathrm{Hg}$. Better correlation could be shown between the difference $\mathrm{ABP}-\mathrm{CCP}$ and CPP $(R=0.57 ; \mathrm{n}=98)$, although the $95 \%$ CI for prediction was still as high as $25 \mathrm{~mm} \mathrm{Hg}$.

The difference between CCP and ICP was significantly $(p<0.0002)$ associated with the state of cerebral autoregulation described by index $\mathrm{Mx}$ (fig $5 \mathrm{~A}$ ).

Similarly, the association between CCP-ICP and cerebral perfusion pressure was highly significant $(\mathrm{p}<0.0001$, fig 5$)$.

\section{Discussion}

The idea of critical closing pressure measurement in head injury proved to be an intriguing concept; however, there is still much uncertainty about its interpretation.

It is difficult to judge which method of CCP calculation is better. Both CCP1 and CCP2 were closely correlated, although in individual patients the absolute difference between these two estimators was as high as $20 \mathrm{~mm} \mathrm{Hg}$. The CCP2 theoretically allows for better accuracy when the arterial pressure transducer used has a limited frequency response, or when arterial pulse pressure, usually measured in the radial or dorsalis pedis artery, differs from the pulse pressure which would be measured in brain basal arteries.

CCP seems to react to time related variations in mean ICP, but individual variability of the CCP makes prediction of ICP unreliable with a $95 \%$ error range of $\pm 27 \mathrm{~mm} \mathrm{Hg}$. Prediction of $\mathrm{CPP}$ using the difference $\mathrm{ABP}-\mathrm{CCP}$ showed a similar prediction error. As the value of CPP is much higher than ICP (over five times higher in this studied group of patients), the estimation of CPP using critical closing pressure seems to be relatively more accurate than an estimation of ICP.

A significant correlation between critical closing pressure and both ICP and ABP indicates that CCP may be useful for monitoring haemodynamically relevant incidences of intracranial hypertension or arterial hypotension.

Whether the difference between CCP and ICP is proportional to cerebrovascular tone still remains problematic. The difference showed a consistent decrease during plateau waves, theoretically caused by arterial vasodilatation. ${ }^{18}$ On the other hand, during refractory intracranial hypertension CCP-ICP usually increased, moreover, the calculated value may become negative.

CCP-ICP decreased significantly when CPP decreased, which may be interpreted as an effect of cerebral vasodilatation. Similarly, CCP-ICP was significantly correlated with the index of autoregulation ( $\mathrm{Mx})$, exhibiting lower values when autoregulation was worse. It can be speculated that disturbed autoregulation in this cohort of patients was predominantly associated with maximal vasodilatation, which could support an early hypothesis ${ }^{10}{ }^{11}$ that the value of CCP-ICP reflects generalised muscle tone in arterial walls. On the other hand, the difference of CCP and ICP did not correlate with clinical outcome after head injury. So far all indices of pressure autoregulation, both derived from TCD as $\mathrm{Mx}^{9}$ and transient hyperaemic response, ${ }^{19}$ from waveforms of ICP and $\mathrm{ABP},{ }^{20}$ or $\mathrm{CO}_{2}$ reactivity ${ }^{21}$ proved to correlate with outcome. Therefore, even if CCP-ICP provides some information regarding the tone of the cerebral arterial bed and, indirectly, cerebral autoregulation, it still lacks clearly defined clinical significance.

Another interesting concept might be that the difference CCP-ICP may express pathological changes of vascular tone such as cerebral vasospasm or vasoparalysis. Unfortunately, the group of patients studied did not include clearly confirmed cases of posttraumatic vasospasm. The reason for that could be purely technical; the TCD ultrasonograph used (PCDop 842, SciMed, Bristol, UK) was unable to record momentary flow velocity $>200 \mathrm{~cm} / \mathrm{s}$. Therefore a section of MCA with undistorted flow velocity pulse waveform was always chosen for insonation.

On the other hand patients with average ICP $>30 \mathrm{~mm} \mathrm{Hg}$, in whom the incidence of vasoparalysis was possibly greater, had CCP-ICP values significantly reduced $(-8$ $\mathrm{mm} \mathrm{Hg}$ for group with $\mathrm{ICP}>30 \mathrm{~mm} \mathrm{Hg}$ versus $10 \mathrm{~mm} \mathrm{Hg}$ for the group with averaged value of ICP $<30 \mathrm{~mm} \mathrm{Hg}(\mathrm{p}<0.00001))$. Similarly significantly lower values of CCP-ICP $(p<0.0001)$ were noted in a group of patients 
in whom transmission of slow waves of $\mathrm{ABP}$ to ICP was shown to have a passive character which may be another independent indicator of vasoparalysis. ${ }^{20}$

\section{Conclusion}

Although critical closing pressure correlates with ICP, CPP, and cerebral autoregulation in patients with head injury, it did not correlate with outcome after head injury. Critical closing pressure reacts sensitively to short lasting waves of ICP and ABP, including plateau and $\mathrm{B}$ waves.

MC, PS, and SP are unpaid leave from Warsaw University of Technology, Poland.

1 Dahl A, Lindegaard KF, Russell D, et al. A comparison of transcranial Doppler and cerebral blood flow studies to assess cerebral vasoreactivity. Stroke 1992;23:15-19.

2 Dahl A, Russell, Nyberg-Hansen R, et al. A comparison of regional cerebral blood flow and middle cerebral artery blood flow velocities: simultaneous measurements in healthy subjects. F Cereb Blood Flow Metab 1992;12:104955.

3 Kontos HA. Validity of cerebral arterial blood flow calculations from velocity measurements. Stroke 1989;20:1-3.

4 Chan KH, Miller DJ, Dearden M, et al. The effect of changes in cerebral perfusion pressure upon middle cerebral artery blood flow velocity and jugular bulb venous oxygen saturation after severe brain trauma. $\mathcal{F}$ Neurosurg 1992;77:55-61.

5 Giller CA, Ratcliff B, Berger B, et al. An impedance index in normal subjects and in subarachnoid hemorrhage. Ultranormal subjects and in subarachn
sound Med Biol 1996;22:373-82.

6 Czosnyka M, Richards H, Pickard JD, et al. Frequencydependent properties of cerebral blood transport: an experimental study in rabbits. Ultrasound in Medicine and Biology 1994;20:391-9.

7 Steinmaier R, Bauhuf C, Hubner U, et al. Slow rhythmic oscillations of blood pressure, intracranial pressure, micro- circulation and cerebral oxygenation: dynamic interrelation and time course in humans. Stroke 1996;27:2236-43.

8 Diehl RR, Linden D, Lucke D, et al. Phase relationship between cerebral blood flow velocity and blood pressure. A clinical test of autoregulation. Stroke 1995;26:1801-4.

9 Czosnyka M, Smielewski P, Kirkpatrick P, et al. Monitoring of cerebral autoregulation in head-injured patients. Stroke 1996;27:829-34.

10 Burton AC. On the physical equilibrium of the small blood vessels. Am F Physiol 1951;164:319-29.

11 Dewey RC, Pieper HP, Hunt WE. Experimental cerebral hemodynamics. Vasomotor tone, critical closing pressure, and vascular bed resistance. Neurosurgery 1974;41:597606.

12 Aaslid R. Cerebral hemodynamics. In: Newell DW, Aaslid R, eds. Transcranial Doppler. New York: Raven Press, 1992: $9-55$.

13 Michel E, Hillebrand S, von Twickel J, et al. Frequency dependence of cerebrovascular impedance in preterm neonates: a different view on critical closing pressure. $\mathcal{F}$ Cereb Blood Flow Metab 1997;17:1127-31.

14 Panerai RB, Kelsall AW, Rennie JM, et al. Estimation of critical closing pressure in the cerebral circulation of newborns. Neuropediatrics 1995;26:168-73.

15 Panerai RB, Coughtrey H, Rennie JM, et al. A model of the instantaneous pressure-velocity relationships of the neonainstantaneous pressure-velocity relationships of the

16 Michel E, Zernikow B, von Twickel J, et al. Critical closing pressure in preterm neonates: towards a comprehensive model of cerebral autoregulation. Neurol Res 1995;17:14955

17 Lindegaard KF, Lundar T, Wiberg J, et al. Variations in middle cerebral artery blood flow investigated with non-invasive transcranial blood velocity measurements. Stroke 1987;18:1025-30.

18 Rosner MJ, Becker DP. Origin and evolution of plateau waves: experimental observations and theoretical model. $\mathcal{F}$ Neurosurg 1984; 60:312-424.

19 Smielewski P, Czosnyka M, Kirkpatrick P, et al. Evaluation of transient hyperemic response test in head injured patients. 7 Neurosurg 1997;86:773-8.

20 Czosnyka M, Smielewski P, Kirkpatrick P, et al. Continuous assessment of the cerebral vasomotor reactivity in head injury. Neurosurgery 1997;41:11-19.

21 Steiger HJ, Aaslid R, Stoos R, et al. Transcranial Doppler monitoring in head injury: relations between type of injury, flow velocities, vasoreactivity and outcome. Neurosurgery 1994;34:79-86 